\title{
Serge Genest
}

Anthropologue, professeur au département d'anthropologie, Université Laval

(1994)

\section{"SIDA et transferts des connaissances"}

Un document produit en version numérique par Jean-Marie Tremblay, bénévole, professeur de sociologie au Cégep de Chicoutimi

Courriel: jean-marie tremblay@uqac.ca

Site web pédagogique : http://www.uqac.ca/jmt-sociologue/

Dans le cadre de la collection: "Les classiques des sciences sociales"

Site web: http://classiques.uqac.ca/

Une collection développée en collaboration avec la Bibliothèque

Paul-Émile-Boulet de l'Université du Québec à Chicoutimi

Site web: http://bibliotheque.uqac.ca/ 
Cette édition électronique a été réalisée par Jean-Marie Tremblay, bénévole, professeur de sociologie au Cégep de Chicoutimi à partir de l'article de :

Serge Genest

Anthropologue, professeur au département d'anthropologie, Université Laval.

\section{"SIDA et transferts des connaissances.”}

Un article publié dans l'ouvrage de Louis-Jacques Dorais, Serge Genest et Louise Mauger, Autour des transferts Orient-Occident. Perspectives anthropologiques, pp. 15-31. Québec: Université Laval, Groupe d'études et de recherches sur l'Asie contemporaine, 1994, 119 pp. Collection : Documents du GÉRAC, no 8.

[Autorisation formelle accordée le 9 novembre 2005 par l’auteur de diffuser ce texte.]

Courriel Serge.Genest@ant.ulaval.ca

Polices de caractères utilisée :

Pour le texte: Times New Roman, 14 points.

Pour les citations : Times New Roman 12 points.

Pour les notes de bas de page : Times New Roman, 12 points.

Édition électronique réalisée avec le traitement de textes

Microsoft Word 2004 pour Macintosh.

Mise en page sur papier format

LETTRE (US letter), 8.5', x 11'’)

Édition complétée le 11 février 2006 à Chicoutimi, Ville de Saguenay, province de Québec. 
Serge Genest

Anthropologue, professeur au département d'anthropologie,

Université Laval.

\section{“SIDA et transferts des connaissances.”}

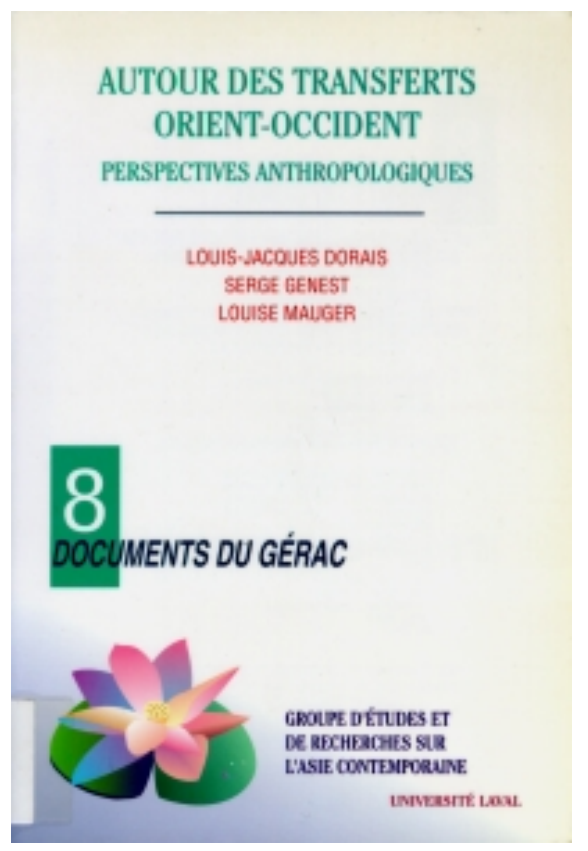

Un article publié dans l'ouvrage de Louis-Jacques Dorais, Serge Genest et Louise Mauger, Autour des transferts Orient-Occident. Perspectives anthropologiques, pp. 15-31. Québec: Université Laval, Groupe d'études et de recherches sur l'Asie contemporaine, 1994, 119 pp. Collection : Documents du GÉRAC, no 8. 


\section{Table des matières}

$\underline{\text { Introduction }}$

Transferts et transferts de connaissances : définitions

Structuration de la perception du sida dans les pays du Nord

Le sida et les pays du Sud

Milieux récepteurs et transferts de connaissances

$\underline{\text { Conclusion }}$

Bibliographie 


\section{Introduction}

$\underline{\text { Retour à la table des matières }}$

Certes, le thème des transferts, même ramené à un ensemble plus « limité » comme celui des connaissances, s'avère fort étendu. Pourtant, malgré cette ouverture thématique, s'interroger sur les transferts de connaissances en matière de sida ne surgit pas à l'esprit comme une évidence.

Toutefois, malgré sa complexité - dans le sens que les systémistes donnent à la notion de complexité (de Rosnay, 1975) - ce sujet s'avère d'une grande pertinence pour réfléchir sur les transferts de façon générale et d'une importance primordiale dans l'examen des rapports Nord/Sud. Par ailleurs, l'anthropologie peut offrir une lecture originale de la construction sociale et culturelle des connaissances, des transferts et du sida.

Après avoir précisé ce qu'il faut entendre ici par «transferts", nous traiterons de la façon dont se sont développées les connaissances sur le sida dans les sociétés du Nord. Cette analyse permettra de montrer ensuite quelles contraintes sociales et culturelles connaissent les pays du Sud et qui influent directement sur les transferts de connaissances sur le sida. 
La Thaïlande, pays en plein essor économique et déjà doté d'infrastructures de santé appréciables, est également aux prises avec une croissance effrénée du nombre de personnes séropositives et sidéennes. En cela, elle réunit plusieurs conditions permettant de mieux comprendre les difficultés associées aux transferts des connaissances en contexte international.

\section{Transferts et transferts de connaissances : définitions}

$\underline{\text { Retour à la table des matières }}$

On ne saurait s'engager dans une réflexion sur les transferts de connaissances à l'échelle internationale sans déterminer, ne serait-ce que minimalement, de quoi nous parlons.

La notion de «transfert » ne devrait s'appliquer, à notre avis, que lorsqu'un récepteur (un individu aussi bien qu'une collectivité) "retient » une « information » (qui se présente sous la forme d'une technologie, i.e. une technique et la manière de l'utiliser, aussi bien que d'une formation ou de toute autre connaissance) transmise par un émetteur. Il n'y a transfert que lorsqu'une transmission réussit. Convenons néanmoins que transfert est équivalent à transmission, comme le langage de tous les jours nous y a habitués.

Il faut aussi s'entendre sur le fait que tout transfert de technologie constitue aussi un transfert de connaissances puisque l'ensemble technique transféré est le produit du développement des connaissances dans le milieu ou cette innovation a vu le jour. Ceci permet d'éviter, nous semble-t-il, de traiter de transfert de connaissances en matière de sida, en pensant que les difficultés rencontrées dans ce contexte ne se présentent pas dans des termes similaires à toute autre forme de transfert. 
Le transfert des connaissances sur le sida s'effectue donc à partir des mêmes contraintes que celles qu'on rencontre dans d'autres contextes. Les difficultés sont toutefois amplifiées parce qu'on aborde ce problème de santé à plusieurs niveaux en même temps : médical et pharmaceutique, de contrôle épidémiologique et de prévention de la maladie. Et il faut se rappeler que c'est à partir d'un contexte social et culturel particulier que les connaissances sur le sida ont vu le jour. Revenons-y brièvement.

\section{Structuration de la perception du sida dans les pays du Nord}

$\underline{\text { Retour à la table des matières }}$

On se souvient que, lorsqu'on a commencé à parler de cette maladie en Amérique du Nord, les homosexuels, les toxicomanes et les Haïtiens furent identifiés comme les principaux porteurs de ce virus. On sait aussi le tollé qui, dès cette époque, s'en est suivi. Une telle identification de « coupables » gênait, quel qu'en ait pu être le fondement par ailleurs.

Lorsque la nouvelle fut lancée dans le public, les laboratoires de recherche médicale étaient déjà au travail dans l'espoir d'identifier le virus, de le mieux connaître et de mettre au point un vaccin pour contrer la maladie. Point n'est besoin d'insister ici sur la progression des travaux de laboratoire au cours des années 80 et les rebondissements et impasses qu'ils ont connus.

Ce qui mérite cependant d'attirer l'attention, ce sont les millions de dollars consacrés à ces travaux et l'orientation technologique très avancée qu'ils affichent. C'est là un premier élément à garder en mémoire au moment de parler des transferts des connaissances du Nord vers le Sud : la sophistication et les coûts de mise au point et d'utilisation aussi bien des médicaments que d'un vaccin potentiel. 
D'autre part, devant ce qu'on voyait déjà se dessiner comme une épidémie, rien d'étonnant à ce que les appareils d'État aient désiré posséder le maximum d'information sur les segments de la population que la maladie atteignait de façon la plus évidente. Les services de santé publique mis sur pied dans les pays du Nord depuis le milieu du siècle dernier offrent cette délicieuse ambiguïté d'être à la fois nécessaires pour assurer le mieux-être des populations tout en devenant un formidable appareil de contrôle dans les mains des autorités politiques (Leclerc, 1979).

Grâce à ces recherches, on a vite constaté que, parmi les personnes identifiées comme séropositives ou encore développant le sida, les homosexuels et les toxicomanes figuraient en tête de liste. Un profil qui se dégageait particulièrement clairement aux États-Unis (Friedman, 1993), mais qui avait également ses répercussions ailleurs dans le monde à travers les médias (Herzlich et Pierret, 1993). Et cette " réalité » médicale et épidémiologique allait permettre de structurer l'imaginaire face à une maladie identifiée par les spécialistes comme le syndrome d'immuno-déficience acquise et dont le sigle, en anglais comme en français, allait passer dans le langage courant.

Comment en effet ne pas penser - et plusieurs auteurs ne s'en sont pas privé - de rapprocher sida et peste ou tuberculose, voire lèpre. Des épidémies qui ont connu leur apogée en d'autres périodes historiques et qui ont également favorisé l'émergence d'explications diverses la plupart du temps éloignées de celles que la biomédecine fournit.

Ces analyses permettent de dégager un certain modèle : la recherche de causes sociales à une maladie qui, par son ampleur même, prend effectivement l'allure d'un problème social autant que de santé !

Dans l'esprit de chaque individu, les « causes sociales » à un mal d'une telle ampleur doivent se matérialiser dans des personnes ou, plus exactement, dans le statut ou le comportement de celles-ci. C'est la recherche des boucs-émissaires. Le sida a connu les siens ! Et c'est aussi sur cette "image » que se sont organisées la perception de la maladie et les interventions pour la prévenir, faute de pouvoir la guérir. 
Lorsqu'est venu le moment de communiquer la connaissance médicale et les analyses épidémiologiques à un public élargi dans les pays du Nord, il a fallu du temps pour redresser certaines idées. Mais au même moment, on transmettait aussi ces nébuleuses de connaissances (pour ne pas dire ces connaissances nébuleuses) ailleurs dans les pays du Sud.

Le texte de Bibeau (1991) montre bien comment l'imprécision des connaissances a conduit à des analyses "scientifiques » où se sont infiltrées toutes sortes d'images, d'impressions d'autant plus dommageables qu'elles émanaient de travaux de recherche prétendant à la rectitude du savoir.

Lors de la prise de conscience de l'ampleur de l'épidémie du sida dans les pays du Nord, et après constat de la place prépondérante qu'y occupaient et les homosexuels et les toxicomanes, assez rapidement un ensemble de perceptions se sont installées et furent véhiculées par les médias. Ce qui a contribué chez nous à associer le sida à des comportements jugés marginaux comme l'homosexualité et l'utilisation des drogues fortes.

Évidemment, cette image a dû se transformer au fil des ans avec la croissance grandissante de cas de sida chez les hétérosexuels, la contamination d'hémophiles par les produits sanguins ou la transmission de la séropositivité à l'enfant par sa mère. Il n'en demeure pas moins que ce sont les données de départ (homosexualité, toxicomanie) qui ont structuré les interventions en matière de prévention et qui continuent de le faire.

A partir du moment où les homosexuels ont compris les dangers qu'ils couraient dans le multipartenariat sexuel et dans leurs pratiques sexuelles de manière plus générale, sans compter le nombre d'amants ou d'amis décédés donnant à la mort par contamination un visage très réel, des changements importants apparurent chez eux (Friedman, 1993). Ce qui fut mis en évidence sur la Côte-Ouest américaine en particulier où les communautés homosexuelles ont réagi promptement et efficacement à ce problème majeur de santé grâce, entre autres, à l'organisation communautaire qui y existait déjà. 
Mais on est loin d'avoir atteint le même succès dans la prévention avec la population hétérosexuelle. Des études se poursuivent dans tous les champs de recherche et d'intervention pour tenter de mieux comprendre les comportements humains face à la menace de contamination par le VIH (Breton, 1993 ; Gariépy et Bertrand, 1989 ; Kegeles, 1988 ; Keller, 1993 ; Levy et Matcka-Tyndale, 1992 ; Sunenblick, 1988, pour ne citer que quelques textes). Et ce qui constitue encore une série d'énigmes ou de problèmes sans solution apparente dans les sociétés du Nord sert de base de transfert des connaissances vers les pays du Sud !

\section{Le sida et les pays du Sud}

$\underline{\text { Retour à la table des matières }}$

Nous avons pu établir que le contexte dans lequel se sont développées les connaissances sur le sida comprenait :

1. une recherche médicale hautement technique et coûteuse ;

2. des analyses épidémiologiques construites sur un appareil administratif et de santé publique très structuré et appuyé de ressources humaines compétentes et en nombre ;

3. des interventions de prévention dirigées vers le comportement sexuel fondées sur l'épidémiologie spécifique de la maladie telle qu'on la retrouve aux États-Unis.

Ce sont ces éléments que l'on désire transférer vers des pays qui sont aux prises avec le sida et qui, soit parce qu'ils n'ont pu en mesurer l'ampleur que récemment, soit parce qu'ils manquent de moyens d'intervenir, acceptent qu'on les aide à combattre ce fléau. 
Mais la littérature sur la transmission des connaissances techniques en particulier est suffisamment abondante et éloquente au sujet des multiples expériences, succès et échecs, qui ont eu cours jusqu'à maintenant, pour qu'on en tire au moins des éléments de réflexion générale.

Celle que propose Callon (1992) à propos de l'innovation technique convient aussi aux transferts, à notre avis. Il ne faut donc pas oublier en premier lieu que la connaissance qu'on a développée dans une société est le produit du travail de plusieurs groupes ou individus, de réseaux « ... qui ont permis à des idées d'être partagées, de se matérialiser dans des dispositifs techniques qui constituent, en quelque sorte, le ciment de ces réseaux ». (p. 14)

Autrement dit, les connaissances biomédicales, épidémiologiques, comportementales et informationnelles mises au point sur le sida dans les sociétés du Nord, en tant qu'« innovations » dans ces sociétés ont une coloration spécifique. Elles sont construites historiquement, socialement et culturellement.

Dans le domaine des technologies en particulier - ce qui inclut la biomédecine - on peut voir tantôt les développements de la recherche technologique (technological push), tantôt la demande de l'industrie (market pull) favoriser l'innovation. Cette dynamique qui caractérise les sociétés du Nord présentement ne se trouve pas dans celles du Sud dans les mêmes termes.

De plus, reconnaissons, avec Callon encore une fois, qu'« un acteur a toujours de bonnes raisons de remettre en cause un choix technique qui ne va pas dans le sens de ses projets, de ses intérêts ou de ses convictions, comme il peut avoir de bonnes raisons de le soutenir ». (p. 12)

Pour qu'une innovation voit le jour, ou pour qu'un transfert de connaissances ait lieu, il faut que les institutions, les groupes ou les individus s'y sentent parties prenante et y trouvent leur intérêt.

Jusqu'à maintenant nous nous sommes arrêté sur les conditions qui ont prévalu dans la structuration de l'analyse du sida et la prévention de la maladie ainsi qu'à quelques conditions générales nécessaires aux 
transferts de connaissances. Il reste maintenant à situer à grands traits les contextes sociaux et culturels dans lesquels s'insèrent les connaissances construites et transmises par les pays du Nord vers ceux du Sud.

\section{Milieux récepteurs et transferts de connaissances}

$\underline{\text { Retour à la table des matières }}$

Bien sûr, la structure administrative de santé publique varie selon les régions, voire les pays, auxquels on s'adresse. Des similitudes existent néanmoins dans les réactions de ces appareils de santé autant que dans les populations. Elles nous permettent d'approcher les problèmes des transferts dans des termes plus généraux qu'au cas par cas.

Commençons par examiner le transfert des connaissances plus directement médicales : v.g. les travaux sur la mise au point d'un vaccin, le développement de médicaments pour ralentir la progression de la maladie chez les personnes atteintes. Étant donné les coûts, l'infrastructure technique et la formation du personnel nécessaires à ces recherches, les transferts de connaissances des pays du Nord vers les pays du Sud se font surtout par les produits que le marché pharmaceutique rend disponibles ou encore par la sélection de malades volontaires pour tester l'efficacité de certains de ces médicaments. Ces connaissances transitent par les médecins des pays du Sud. Les problèmes qui vont surgir à ce niveau sont d'ordre éthique lorsqu'il s'agit de tester des médicaments et financier pour pouvoir offrir les « services » mis au point dans les pays du Nord.

Les transferts effectués vers la Thaïlande à partir des États-Unis, par exemple, sont précisément de ces deux types. D'une part, les services de santé acceptent et/ou achètent les produits pharmaceutiques disponibles sur le marché pour venir en aide aux personnes atteintes 
de sida. D'autre part, les autorités administratives et médicales pourraient envisager de tester de nouveaux médicaments sur des personnes atteintes du virus, entre autres dans l'armée.

Bien qu'on ne puisse considérer les difficultés liées à ces transferts comme des questions mineures, elles demeurent relativement cernables d'une part et montrent déjà, d'autre part, que ce sont les préoccupations des pays du Nord qui orientent les termes de ces transferts de connaissances.

Voyons maintenant ce qui concerne les recherches épidémiologiques. Bien sûr, en matière de santé publique, la capacité à cerner l'ampleur d'un problème de santé ou d'une épidémie s'avère essentielle pour intervenir. Dans les pays du Nord, des ressources humaines et financières sont systématiquement consacrées à ces analyses. De plus, les appareils d'État qui sont en place assurent le déroulement de tels travaux dans des conditions optimales.

Le transfert de connaissances techniques par la formation disciplinaire ne rencontrera pas d'obstacle majeur ici non plus si la structure administrative et de gestion qui supporte la collecte des informations et leur analyse peut répondre aux demandes. On sait que le simple acheminement de données quantifiables sur le sida à partir du réseau des institutions de santé dans certains pays du Sud peut présenter des difficultés presque insurmontables dans ces pays.

On aurait tort, dans ces conditions, de penser que la seule formation, la seule transmission des connaissances techniques appropriées, suffira à développer une couverture épidémiologique adéquate. La réussite de tels transferts est conditionnée, entre autres, par la qualité des appareils de santé en place dans le pays visé et par une compréhension par le personnel affecté à ces tâches des enjeux d'une bonne analyse épidémiologique.

Sans entrer dans les détails de l'organisation des soins de santé de base en Thaïlande, on peut toutefois noter que le pays s'est doté depuis quelques années d'un système pyramidal d'intervention relativement efficace qui va du ministère de la Santé jusqu'au niveau des regroupements de villages (Silcock, 1977 ; Semsri, 1989). 
De plus, bien qu'il y ait encore place pour l'amélioration des ressources humaines, l'État thaïlandais a consenti des efforts notables dans la formation du personnel médical et aussi dans celle des spécialistes de l'analyse sociale en santé publique. Mais, malgré des conditions générales qui facilitent la transmission des modèles de surveillance épidémiologique et une structure organisationnelle au fonctionnement relativement adéquat, on ne saurait dire que la Thaïlande possède tous les atouts pour que la transmission des connaissances en matière de sida provenant des États-Unis, par exemple, se fasse sans problème. Et pourtant, redisons-le, il s'agit d'un pays relativement bien outillé pour absorber les connaissances que les pays du Nord développent.

Toutefois, même si la transmission des connaissances des pays du Nord vers ceux du Sud présente des difficultés qu'on aurait tort de négliger, toutes proportions gardées, ce n'est pas àces niveaux que les défis majeurs apparaissent. Le vaste domaine de la prévention du sida pose en fait les problèmes majeurs.

Il a bien fallu admettre que la seule issue réaliste devant la montée du sida dans les pays du Nord était de travailler à informer la population sur les conditions de transmission de la maladie et d'inciter les personnes dites «à risque " d'adopter des comportements de prévention. C'est ce qu'on peut qualifier « d'approche communicationnelle».

Centrée au départ sur certains segments de la population, il a bientôt fallu reconnaître qu'il n'y avait plus que les toxicomanes, les homosexuels ou les prostitué-e-s qui étaient «à risque », mais que toute la population sexuellement active « risquait » également la contamination. Comme on le sait, ce sont les conditions du développement du sida dans les pays du Sud qui, en particulier, ont permis d'effectuer une telle prise de conscience. Désormais, le sida n'est plus uniquement associable à la marginalité ; toute personne sexuellement active doit se protéger de la maladie.

Force est toutefois de constater que le discours sur la prévention développé dans les sociétés du Nord ne s'est avéré que partiellement adapté à la réalité qu'elles-mêmes connaissaient. En effet, sur la base 
des constatations épidémiologiques, les campagnes d'information et de prévention se sont concentrées au départ sur les groupes dits «à risque ».

Malgré les progrès laborieux du début, on peut dire que les mesures de prévention : utilisation de condoms, de seringues stériles ont fait leur chemin. Mais il a bien fallu reconnaître que les adolescents, les adultes devraient aussi modifier leurs comportements.

S'il fut possible d'obtenir quelque succès avec les homosexuels, peut-être avec les prostitué-e-s, et éventuellement avec les toxicomanes, on constate qu'il en va autrement avec les autres segments de la population. On tente encore maintenant de s'expliquer les raisons qui font que, malgré une information soutenue sur les dangers de rapports sexuels sans préservatif, les adolescent-e-s de nos sociétés continuent d'afficher toutes sortes de réticences ou de blocages sur l'utilisation du condom (Breton, 1993).

On répète encore ces jours-ci que le préservatif demeure, à toute fin utile, le seul moyen de se prémunir contre le VIH pour le moment. Pendant ce temps, des millions de gens refusent, pour des motifs varies, d'aseptiser leur sexualité, de renoncer à la reproduction, ou de transformer leur émotion amoureuse en rationalité préventive. Et ce sont ces « connaissances » que nous voulons transférer vers les pays du Sud !

Faut-il dès lors s'étonner que les campagnes de prévention du sida ne reçoivent qu'un accueil sceptique dans les pays africains ou asiatiques qui sont pourtant durement touchés par l'épidémie ? Comment faire admettre à des personnes pour qui la reproduction constitue l'un des fondements essentiels de la vie (v.g. en Afrique noire) qu'il faut faire usage du condom ! Comment susciter l'enthousiasme pour l'abstention de rapports sexuels en dehors des liens matrimoniaux dans des populations qui ont une vision libérale en cette matière depuis des centaines d'années! Les travaux récents sur la propagation du sida en Thaïlande reviennent maintenant constamment sur cette "donnée de base » des rapports sexuels dans cette société (Muecke, 1992 ; Bamber, 1993 ; Hewison et Underwood, 1992). 
Comment expliquer à tout ce monde que ce n'est rien si on n'associe plus rapport émotionnel, rapport sexuel et reproduction puisque la technologie nous permet désormais de nous reproduire artificiellement !

Ce n'est certes pas parce qu'on prêtera une attention accrue à la langue, au symbolisme approprié dans la communication des messages de prévention du sida qu'il faut espérer un transfert harmonieux des connaissances. Les pays du Nord n'ont pas encore fait pour euxmêmes l'examen complet de la situation. Ils ont conservé une vision qui correspond davantage à une perception du sida par groupes «à risque ». Or, cette façon de voir n'est que partiellement adaptée à l'imaginaire de l'ensemble de la population. Et on souhaiterait qu'il y ait transmission réussie, i.e. transfert de connaissances des programmes d'éducation, d'information et de communication?

\section{Conclusion}

$\underline{\text { Retour à la table des matières }}$

Traditionnellement, l'anthropologue s'intéresse à l'analyse des rapports sociaux dans les sociétés non occidentales. Son expertise se situe donc dans une connaissance de première main de ces sociétés, dans l'analyse comparative qui peut s'en suivre et qui conduit souvent à une compréhension différente de ce qui se passe dans nos propres sociétés.

Ce sont les contacts avec les pays du Sud qui auront permis de donner un nouveau visage à l'épidémie du sida : sa présence chez les personnes hétérosexuelles, dont beaucoup de femmes, et l'obligation consécutive de modifier progressivement le modèle d'interprétation de l'épidémie pour y inclure les comportements à risque, par exemple.

Mais il faut aussi regarder les conditions du développement des connaissances sur le sida dans les pays du Nord parce qu'on ne saurait transférer quelque information que ce soit sur cette question si nous ne 
prenons d'abord conscience que ce que nous transmettons constitue en fait une vision particulière du sida avec les moyens d'intervention que nous lui associons. De plus, le développement de ces connaissances s'effectue à partir de nos préoccupations : une dynamique scientifique spécifique, orientée par la recherche de solutions de type technologique aux problèmes de santé et un ensemble de rapports caractéristiques entre l'appareil d'État et les pressions du secteur privé.

Lorsqu'on analyse les transferts de connaissances sous cet angle, une analogie surgit aisément à l'esprit : l'implant chez les humains. Que de précautions ne faut-il prendre ! Que de réactions imprévues ! Et on voudrait qu'il soit plus simple d'agir sur les comportements humains, sur les sociétés qui sont tellement plus complexes! Les trois domaines de connaissances les plus sollicités face au sida : médecine, épidémiologie et communication, ont servi ici à montrer cette complexité.

Devant les transferts de connaissances sur le sida, l'anthropologue peut s'autoriser de son expertise pour dire essentiellement deux choses :

1. On ne parviendra à transmettre avec succès nos connaissances que si nous prenons en considération l'ensemble des institutions et des rapports sociaux et culturels qui font l'objet de ces transferts.

2. Il n'est pas possible de tenir compte de la dynamique des sociétés du Sud si nous ne reconnaissons pas que nos connaissances sur le sida se construisent d'abord et avant tout à partir de nos intérêts et de notre vision de cette épidémie. 


\section{Bibliographie}

$\underline{\text { Retour à la table des matières }}$

BAMBER, S. D., K. J. HEWISON et P. J. UNDERWOOD, «A History of Sexually Transmitted Diseases in Thailand: Policy and Politics ». Genitourin Med, 69, 1993, pp. 148-157.

BIBEAU, G., « L'Afrique, terre imaginaire du sida. La subversion du discours scientifique par le jeu des fantasmes ». Anthropologie et sociétés, 15, 2-3, 1991, pp. 125-147.

BRETON, D., Conception de la sexualité et prévention du sida chez des cégépien-ne-s hétérosexuel-le-s de la région de Québec. Québec, Université Laval, mémoire de Maîtrise en anthropologie, 1993, 110 p.

CALLON, M., "Construction des objets techniques : application de la sociologie de la traduction au cas des innovations médicales ». In MOATTI, J.-P. et C. MAWAS (eds.), Évaluation des innovations technologiques et décisions en santé publique. Paris, INSERM, 1992, pp. 7-21.

DE ROSNAY, E., Le macroscope. Paris, Éditions du Seuil, 1975, $303 \mathrm{p}$.

FREIDMAN, S.R., "AIDS as a Sociohistorical Phenomenon. In ALBRECHT, G. and R. ZIMMERMAN (eds), The Social and Behavioral Aspects of AIDS, JAI Press Inc., Advances in Medical Sociology, 3, 1993, pp. 19-36. 
GARIEPY, A. et I. BERTRAND, Le sida et les jeunes. Enquête sur la sexualité des jeunes. Montréal, Éditions Fides,

1989, 124 p.

HERZLICH, C. et J. PIERRET, « From Epidemic to Modem Illness. The Social Construction of AIDS in France ». In ALBRECHT, G. et R. ZIMMERMAN (eds), The Social and Behavioural Aspects of AIDS, JAI Press Inc, Advances in Medical Sociology, 3, 1993 , pp. 59-76.

KEGELES, S. M. et al., "Sexually Active Adolescents and Condoms: Changes over One Year in Knowledge, Attitudes and Use ». American Journal of Public Health, 78, 4, 1988, pp. 460-461.

KELLER, M. L., «Why Don't Young Adults Protect Themselves Against Sexual Transmission of HIV ? Possible Answers to a Complex Question-. AIDS, Education and Prevention, 5, 3, 1993, pp. 220233.

LECLERC, G., L'observation de l'homme. Une histoire des enquêtes sociales. Paris, Editions du Seuil, 1979, 363 p.

LEVY, J. J. et E. MATICKA-TYNDALE, Sexualité, contraception et sida chez les jeunes adultes. Variations ethno-culturelles. Montréal, Éditions du Méridien, 1992, 258 p.

MUECKE, M.A., « New Paradigms for Refugee Health Problems ». Social Science and Medicine, 35, 4, 1992, pp. 515-523.

SERMSRI, S., « Utilization of Traditional and Modem Health Care Services in Thailand ». In QUAH, S. R. (ed), The Tiumph of Practicality, Singapore, Institute of Southeast Asian Studies, 1989, pp. 160179.

SILCOCK, T. H. (ed), "Thailand : An Indigenous Professional Structure ? ». In Professional Structure in South East Asia, Canberra, The Australian National University, 1977, pp. 54-119.

9. 
SUNENBLICK, M. B., "The AIDS Epidemic: Sexual Behavior of Adolescents. Smith College Studies in Social Work, 59, 1, 1988, pp. 21-37. 